\title{
Bio-inspired soft polystyrene nanotube substrate for rapid and highly efficient breast cancer-cell capture
}

\author{
Xueli Liu ${ }^{1,2}$, Li Chen ${ }^{1,2}$, Hongliang Liu ${ }^{1}$, Gao Yang ${ }^{1,2}$, Pengchao Zhang ${ }^{1,2}$, Dong Han $^{3}$, Shutao Wang ${ }^{1}$ and \\ Lei Jiang ${ }^{1}$
}

The softness of materials is very important in cellular processes, such as cell adhesion, spread, proliferation and differentiation. Using soft materials benefits many biological studies and applications, such as wound healing, tissue engineering and clinical surgery. However, in other areas, such as rare cancer-cell capture and isolation, the importance of the softness of materials has not yet been studied. Extracellular matrix suitable for cell contact and survival is formed from soft materials with specific molecules and nanostructures. Herein, we report a soft polystyrene nanotube substrate inspired by the cell microenvironment that achieves rapid and highly efficient breast cancer-cell capture from whole-blood samples with high cell viability by integrating the soft nature of polystyrene polymer with specific capture agents and surface structures. This study provides a potentially optimal candidate for a high-quality breast cancer-cell detection platform and will provide new prospects for designing cell-material interfaces for advanced cell-based biological studies and clinical applications in the future. NPG Asia Materials (2013) 5, e63; doi:10.1038/am.2013.43; published online 20 September 2013

Keywords: bio-inspired; cancer diagnosis; cell capture; nanostructures; polystyrene; soft polymers

\section{INTRODUCTION}

Cells are made from soft materials and live in a microenvironment formed by soft materials. ${ }^{1}$ The softness of synthetic materials greatly influences cellular processes, ${ }^{2-4}$ such as cell adhesion and spread, ${ }^{5}$ stem-cell proliferation and differentiation, ${ }^{6-8}$ and even tumor generation. ${ }^{9}$ Soft materials with low stiffness or tissue-like stiffness significantly benefit normal cell activities and functions. ${ }^{10}$ For example, substrates with extracellular matrix-like stiffness optimally promote the branch regeneration of neurons, ${ }^{11}$ as well as the myotube differentiation of muscle cells. ${ }^{12}$ Many soft materials have been developed for biological studies and applications. Soft polymers such as poly(methyl methacrylate) and polyamides have long been used for medical purposes, such as contact lenses and clinical surgeries. ${ }^{13}$ In recent years, polymer hydrogels have also been explored for their biological value in areas such as wound healing and tissue engineering. ${ }^{14}$ However, in cell-related diagnosis areas, such as drug delivery and rare cell isolation, ${ }^{15-19}$ the importance of the softness of materials interacting with cells has not yet been explored.

The capture and isolation of rare cells, such as circulating tumor cells (CTCs), from the blood of cancer patients are very important for cancer diagnosis and treatment and related biological study. ${ }^{20,21}$ Traditional CTCs isolation methods are mainly based on cell density, size and surface specific molecules and lack specificity and efficiency. In previous research, we have fabricated threedimensional silicon nanowire ( $\mathrm{Si} N \mathrm{NW})$ substrates modified with specific capture agents, showing the advantage of using nanostructures for highly efficient CTCs isolation. ${ }^{22,23}$ Many nanostructured cell-capture substrates have been developed, such as gold cluster-coated $\mathrm{Si} \mathrm{NWs}{ }^{24}$ quartz nanowire arrayss ${ }^{25}$ and electrospun $\mathrm{TiO}_{2}$ nanofibers. ${ }^{26}$ However, those substrates are all based on inorganic materials, which are very rigid and may not be optimal materials for cell capture. A conducting polymer nanodot substrate has been reported for cell capture. ${ }^{27}$ It was found that although nanodots had lower aspect ratios than Si NWs, which reduced the topographical interactions between cell and substrate, the nanodots' substrate still obtained high capture efficiency. This result may be due to the lower softness of conducting polymer compared with inorganic silicon, but the mechanism is not clear. Therefore, it remains necessary to study the influence of softness and the design of soft material-based cell-capture substrates on cell capture.

Currently, along with the development of nanostructured cellcapture systems, in vitro cell-capture material design strategies focus on the combination of both chemical (specific ligand/receptor recognitions) and physical (suitable micro- or nano-topographical interactions) aspects. ${ }^{17-19,22-26}$ However, in vivo extracellular matrix interacting with cells involves chemical, physical and mechanical (including softness) factors. Biological systems in nature have inspired the development and application of many advanced functional materials. ${ }^{28-31}$ Exploring the integration of soft materials with these chemical and physical attributes to mimic the natural cell

\footnotetext{
${ }^{1}$ Beijing National Laboratory for Molecular Sciences (BNLMS), Key Laboratory of Organic Solids, The Institute of Chemistry, Chinese Academy of Sciences, Beijing, China; ${ }^{2}$ Graduate School of Chinese Academy of Sciences, Beijing, China and ${ }^{3}$ National Centre for NanoScience and Technology, Beijing, China

Correspondence: Professor S Wang, Beijing National Laboratory for Molecular Sciences (BNLMS), Key Laboratory of Organic Solids, The Institute of Chemistry, Chinese Academy of Sciences, Zhongguancun North First Street 2, 100190 Beijing, China.

E-mail: stwang@iccas.ac.cn
}

Received 22 March 2013; revised 14 June 2013; accepted 30 June 2013 
microenvironment will be instructive for realizing a high-quality cell-capture platform. Herein, for a proof-of-concept study, we developed soft polystyrene (PS) nanotube (PS NT) substrates that achieved rapid and highly efficient breast cancer-cell capture from whole-blood samples with high cell viability; these substrates were developed by integrating the soft nature of PS polymer with specific capture agents and surface structures (Figure 1). The prepared soft PS NT substrate reached $\sim 80 \%$ cell-capture efficiency after specific antibody modification, which was $\sim 30 \%$ higher than that of the smooth PS substrate. Compared with the inorganic rigid Si NWs cellcapture system we reported previously ( $\sim 40-60 \%$ capture efficiency), ${ }^{22}$ the cell-capture efficiency of our soft PS NT substrate is significantly higher. Our attempt suggests that soft materials are more suitable breast cancer-cell capture substrates compared with rigid materials. They provide a new candidate for a rare cell isolation platform and bring novel views to the design of cell-material interfaces for high-quality biological study and clinical detection in the future.

\section{EXPERIMENTAL PROCEDURE}

\section{Materials}

PS grains were obtained from Sigma-Aldrich Co. (Shanghai, China). (average $M_{\mathrm{w}} \sim 350000$, average $\left.M_{\mathrm{n}} \sim 170000\right)$. Smooth PS sheets were purchased from Zhejiang Plasmed Medical Technology Co., Ltd. (Taizhou, China). Anodic aluminum oxide (AAO) template with pore diameter of $0.2 \mu \mathrm{m}$ was obtained from Whatman. Dimethylbenzene (AR) was purchased from Beijing Chemical Reagent Co. (Beijing, China). Phosphate buffer solution (PBS) was obtained from Hyclone (Logan, UT, USA). 4',6-Diamidino-2-phenylindol (DAPI), acridine orange (bioreagent, for molecular biology) and propidium iodide (PI, $\geqslant 94.0 \%)$ were obtained from Sigma-Aldrich Co. ImmunoPure. DiD fluorescent dye was purchased from Invitrogen (Carlsbad, CA, USA). PE-labeled anti-cytokeratin (CK) and fluorescein isothiocyanate (FITC)labeled anti-CD45 were obtained from BD Biosciences (Franklin Lakes, NJ, USA). Primary antibody (anti-vinculine mouse monoclonal), secondary antibody (FITC-conjugated goat anti-mouse) and TRITC-conjugated Phalloidin were purchased from Sigma-Aldrich. Biotinylated bovine serum albumin (biotin-BSA) was purchased from Thermo Scientific (Waltham, MA, USA). Streptavidin (SA) and biotinylated anti-human epithelial cell adhesion molecule (EpCAM)/TROP1 antibody (Goat IgG) (biotinylated anti-EpCAM) were obtained from R\&D systems (Minneapolis, MN, USA). The breast cancer-cell line (MCF7), prostate cancer cell line (PC3), bladder cancer cell line (T24), cervical cancer cell line (Hela), lymphocyte cell line (Jurkat T) and lymphoma cell line (Daudi) were purchased from Beijing Xiehe, School of Medicine, Cell Resource Center. Male Wistar mice (300-320 g) were purchased from Vital River Laboratory Animal Center (Beijing, China).

\section{Procedures}

Fabrication of PS nanotube substrate. Commercial PS grains (5g) were dissolved in dimethylbenzene $(15 \mathrm{ml})$ to form PS solution. PS solution
( $0.5 \mathrm{ml})$ was dripped onto $1.5 \times 1.5 \mathrm{~cm}^{2}$ glass slide. Then, an AAO template was carefully placed on the surface of the PS solution. Due to capillary force, the PS solution was sucked into the pores of the AAO template. After $48 \mathrm{~h}$, the dimethylbenzene solvent was volatilized completely, and the PS solution turned to solid PS. Finally, the sample was immersed in $\mathrm{NaOH}$ solution $\left(2 \mathrm{moll}^{-1}\right)$ for $15 \mathrm{~min}$ to etch away a layer of AAO and expose the PS nanotubes.

Surface modification of the PS substrates for cell capture. The PS substrates were immersed in biotin-BSA solution $\left(0.1 \mathrm{mg} \mathrm{ml}^{-1}\right.$ in PBS) to modify biotin$\mathrm{BSA}$ on them (room temperature (RT) $2 \mathrm{~h}$, or $4{ }^{\circ} \mathrm{C}$ overnight). After three washes using PBS, the substrates were immersed in SA solution $\left(0.1 \mathrm{mg} \mathrm{ml}^{-1}\right.$ in PBS) for $30 \mathrm{~min}$ (RT), washed with PBS three times, immersed in biotinylated anti-EpCAM solution $\left(10 \mu \mathrm{g} \mathrm{ml}^{-1}\right.$ in PBS) for $30 \mathrm{~min}$ (RT), and then rinsed with PBS three times.

Cell-capture procedure. Anti-EpCAM-modified PS substrates were placed into six-well cell culture plates. Then, cell suspensions ( $3 \mathrm{ml}, 10^{5}$ cells per $\mathrm{ml}$ ) were carefully added into each well and incubated in incubators $\left(37^{\circ} \mathrm{C}, 0.5 \%\right.$ $\mathrm{CO}_{2}$ ) for the predetermined capture time. Afterwards, the substrates were taken out of the cell suspensions and rinsed carefully five times with PBS. After the cells were fixed using paraformaldehyde solution (4 wt $\%$ in PBS), penetrated using Triton-X100 (0.2 wt\% in PBS), and dyed using DAPI solution $\left(2 \mu \mathrm{g} \mathrm{ml}^{-1}\right.$ in water), the substrates were imaged using fluorescence microscopy (Nikon, Ti-E, Tokyo, Japan). After cell imaging, we analyzed and calculated how many cells were captured on the substrate using cell-counting software. Then, by comparing that number with the total number of cells added, we calculated the capture efficiency.

Immunofluorescent staining for actin cytoskeleton and focal adhesions (FAs) of cells. After $45 \mathrm{~min}$ of incubation, MCF7 cells captured on the anti-EpCAMmodified PS substrates were fixed in $4 \%$ paraformaldehyde (in PBS) for $20 \mathrm{~min}$, permeabilized with $0.3 \%$ Triton-X100 (in PBS) for $5 \mathrm{~min}$ and then blocked with 2\% BSA (in PBS) for $30 \mathrm{~min}$ at RT. After removing the block solutions (without washing), cells were incubated with the primary antibody (anti-vinculin mouse monoclonal, diluted 1:400) for $1 \mathrm{~h}$ (RT). After washing with PBS three times (5-10 min each), cells were incubated with the secondary antibody (FITC-conjugated goat anti-mouse, diluted 1:32) for $30 \mathrm{~min}$ (RT). Then, the actin cytoskeleton was stained using TRITC-conjugated Phalloidin ( $15 \mu \mathrm{g}$ in $250 \mu \mathrm{l}$ methanol and diluted 1:100 using $2 \%$ BSA) for $30 \mathrm{~min}$ (RT). After washing with PBS three times (5-10 min each), cells were stained with DAPI $\left(10 \mu \mathrm{g} \mathrm{ml}^{-1}\right.$ in water) for $10 \mathrm{~min}$. Finally, after three washes, the samples were stored in the dark in PBS $\left(4^{\circ} \mathrm{C}\right)$.

Cell capture from artificial whole-blood samples. Artificial whole-blood samples were prepared by spiking cancer cells (MCF7, pre-stained with DiD red dye) in mouse blood at concentrations of 20, 50, 100 and 250 cells per ml. One anti-EpCAM-modified PS NT substrate was placed into one pore of a 12-well culture plate to ensure that the entire base area of the pore was covered by the PS substrate, and $1 \mathrm{ml}$ whole-blood sample was added into the pore for cell capture $\left(37^{\circ} \mathrm{C}, 20 \mathrm{~min}\right)$. Then, the PS substrate was gently washed with PBS five times and taken for fluorescence imaging.

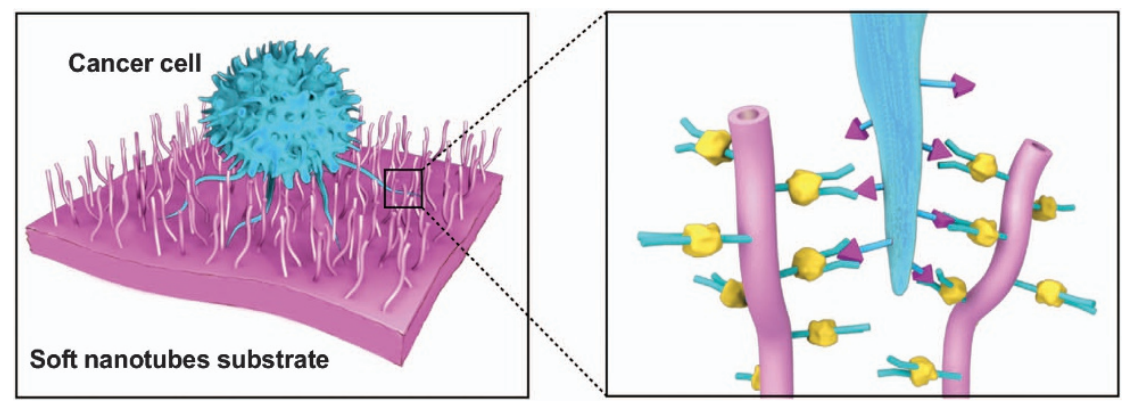

Figure 1 Schematic illustration of cancer-cell capture principle of the soft nanotube substrate. Highly efficient cancer-cell capture is achieved on the substrate by integrating the soft nature of polystyrene (PS) polymers with specific capture agents and surface structures. 
Cells captured on the substrate were identified by a typical three-color immunostaining method. After fixation, permeabilization and blocking, cells were stained with a mixed PE-labeled anti-CK and FITC-labeled anti-CD45 solution $\left(10 \mu \mathrm{ml}^{-1}\right.$ each) at $4{ }^{\circ} \mathrm{C}$ overnight in the dark, washed with PBS three times (5-10 min each) and incubated with DAPI $\left(10 \mu \mathrm{g} \mathrm{ml}^{-1}\right.$ in water) for $5 \mathrm{~min}$. After three washes, the sample was taken for fluorescence imaging.

Instruments and characterization. A field-emission scanning electron microscope (JSM-6700F, JEOL, Tokyo, Japan) was used for characterization of the surface morphologies of the as-prepared PS substrates. The stiffness of the substrates was measured using a nano-indenter (HYSITRONTM, TI950 TriboIndenter). The morphologies of cells captured on the substrates were acquired using an environmental scanning electron microscope (FEI Quanta 200, FEI, Hillsboro, OR, USA). After $45 \mathrm{~min}$ of incubation, cells captured on PS substrates were fixed using glutaraldehyde solution, dehydrated in gradient ethanol and finally dried in critical carbon dioxide for environmental scanning electron microscope observation.

\section{RESULTS AND DISCUSSION}

Fabrication of anti-EpCAM-modified PS nanotube substrate for cell capture

Among the numerous polymers commonly used, we chose PS to fabricate the nanostructured substrate, as PS is biocompatible and widely used in commercial cell culture substrates and biological study materials. We believe that our studies will support new uses for PS materials in cancer diagnosis in addition to their traditional roles. The PS NT substrate was fabricated using a replication method with $\mathrm{AAO}$ as the template (Figure $2 \mathrm{a}){ }^{32}$ By etching AAO away using alkali solution for $15 \mathrm{~min}$, we obtained the PS nanotube array-covered substrate, as shown in Figure 3d. The diameters of the nanotubes were $\sim 200 \mathrm{~nm}$. Then, the as-prepared PS NT substrate was coated with a layer of specific agent (anti-EpCAM, EpCAM antibody) to capture target MCF7 cancer cells (breast cancer cell lines, overexpress EpCAM antigen) ${ }^{33}$ by three modification steps (Figure $2 b$ ). ${ }^{34}$ First, biotinylated BSA (biotin-BSA) was physically adsorbed on the PS NT substrate. Then, a linker SA was conjugated to the substrate through biotin-SA interactions. Finally, biotinylated anti-EpCAM was connected to the PS NT substrate before the cell-capture experiment. The introduction of the BSA molecule here has two advantages: first, due to the firm adsorption of BSA to materials through hydrophobic interactions, the modification process of anti-EpCAM becomes less cumbersome than the traditional NHS/maleimide chemistry method, ${ }^{22}$ and second, the blocking effect of BSA on the substrate could provide an inert surface that prevents the non-specific molecular adsoption and cell adhesion ${ }^{35,36}$ that favor cell-capture specificity.
Cell-capture performance of the functionalized PS NT substrate Cell-capture experiments of the anti-EpCAM-modified PS NT substrates were then carried out by immersing the substrate in MCF7 cell suspensions $\left(3 \mathrm{ml}, 10^{5}\right.$ cells per $\mathrm{ml}$, in a six-well cell culture plate), followed by incubation at $37{ }^{\circ} \mathrm{C}$ in $5 \% \mathrm{CO}_{2}$ for $45 \mathrm{~min}$. For comparison, anti-EpCAM-modified smooth PS substrate (Figure 3a) was also prepared for the cell-capture experiment. After capture, the cells that were immobilized on these substrates were fixed, penetrated, and dyed for imaging and counting using fluorescence microscopy. The results show that the cell-capture efficiency of the anti-EpCAM-modified PS NT substrate was very high, $\sim 80.4 \%$ (Figure $3 \mathrm{e}$ ), and $\sim 30 \%$ higher than that of the smooth substrate ( $\sim 52.6 \%$, Figure $3 b)$.

To investigate the influence of softness on cell capture in more detail, the stiffness of the as-prepared smooth PS and PS NT substrates was measured using a nano-indenter. ${ }^{37}$ For comparison, smooth silicon (smooth $\mathrm{Si}$ ) and Si NW substrates were fabricated according to our previous study using Si NWs for cell capture, ${ }^{22}$ and the stiffness of these two substrates was measured simultaneously. The results show that the stiffness of the smooth PS substrate $\left(\sim 5.82 \times 10^{6} \mathrm{kPa}\right)$ was 28 times lower than that of the smooth $\mathrm{Si}$ substrate $\left(\sim 163.29 \times 10^{6} \mathrm{kPa}\right)$. Similarly, the PS NT substrate $\left(\sim 0.53 \times 10^{6} \mathrm{kPa}\right)$ was $\sim 9$ times softer than the Si NW substrate $\left(\sim 4.74 \times 10^{6} \mathrm{kPa}\right)$. In our previous study, the cell-capture efficiency of the smooth Si and Si NW substrates was $\sim 4-14 \%$ and $45-65 \%$, respectively. ${ }^{22}$ The comparison shows that as the stiffness decreases, the cell-capture efficiency increases. The capture efficiency of our smooth PS substrate was significantly higher than that of the smooth Si substrate, and the capture efficiency of our PS NT substrate was considerably higher than that of the Si NW substrate. ${ }^{22}$ These results indicate that soft PS substrates can obtain higher cell-capture efficiency than rigid Si substrates, revealing that soft materials are more suitable for cell-capture substrates than rigid materials.

\section{Topographical interactions between cells and PS NT substrates}

The morphologies of cells captured on the as-prepared PS substrates were then observed using an environmental scanning electron microscope. The results demonstrated that cells on the smooth PS substrate spread and exhibited a predominantly flat morphology (Figure 3c, supporting information Supplementary Figure S1). In contrast, cells on the PS NT substrate only spread slightly (Figure 3f). Although cells on the smooth PS substrate have protruding surface structures (including filopodia and lamellipodia), there was almost no topographical interaction between cells and the substrate because the substrate surface is smooth (Supplementary Figure S2a). By contrast,
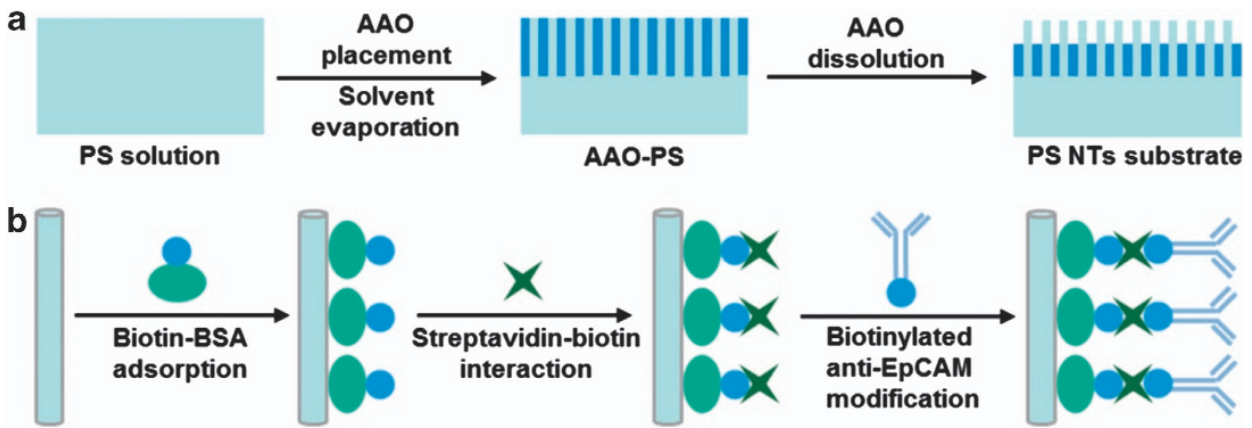

Figure 2 Fabrication and modification of the polystyrene (PS) nanotube (NT) substrate. (a) Fabrication process of the PS NT substrate using the replication method with anodic aluminum oxide (AAO) as the template. (b) Surface modification process to obtain the anti-epithelial cell adhesion molecule (EpCAM)modified PS NT substrate. 

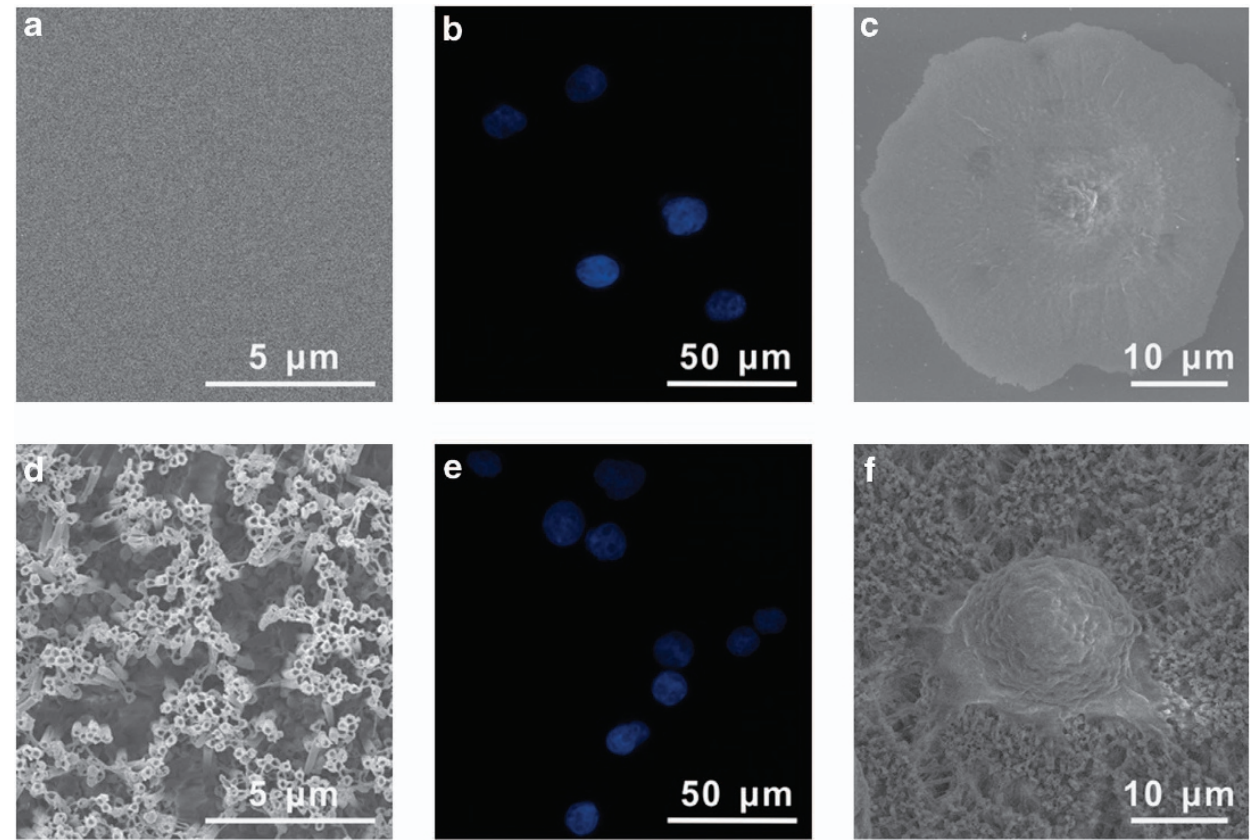

Figure 3 Comparison of the cell-capture efficiency of anti-epithelial cell adhesion molecule (EpCAM)-modified smooth polystyrene (PS) and polystyrene nanotube (PS NT) substrates. (a,d) Scanning electron microscope (SEM) images of the smooth PS and PS NT substrates. (b,e) Corresponding fluorescence images of cells captured on these substrates ( $\times 40$ objective lens). The PS NT substrate exhibited an $\sim 30 \%$ increased cell-capture efficiency compared with the smooth substrate. (c,f) Environmental scanning electron microscope (ESEM) images of cells captured on these two substrates. Cells spreads greatly on the smooth PS substrate without topographical interactions. In contrast, cells spread slightly and protrude filopodia to contact the surface structures of the PS NT substrate.

the surface structures of cells on the PS NT substrate interacted with the nanotubes on the substrate surface, showing enhanced topographical interaction (Figure 3f and Supplementary Figure S2b). This difference in cell-substrate topographical interactions explains the increased cell-capture efficiency on the nanostructured PS substrate compared with the corresponding smooth substrate and demonstrates the topographical enhancement effect on cell capture. ${ }^{17,22}$ In addition, due to the confinement of the substrate structures, cells spread less on the PS NT substrates than on the smooth substrates. Importantly, compared with previously reported inorganic smooth substrates, on which cells maintain a round shape without spreading, ${ }^{22,26}$ our smooth PS substrate greatly enhanced cell spreading. Thus, the PS material has a higher affinity to cells that promote cell attachment and spreading compared with inorganic materials. This higher affinity is most likely due to the soft nature of the PS material, which results in higher cell-capture efficiency. Thus, by integrating the soft nature of PS materials with specific capture agents and surface structures, the soft PS NT substrate has demonstrated its potential for achieving highly efficient cancer-cell capture.

To further explore the topographical interactions between cells and PS substrates, immunochemistry experiments were performed to observe the actin cytoskeleton, the filopodia and lamellipodia and the FAs of cells. Previous studies indicate that cells may sense and respond to substrate topography by mechanotransduction through an integrin-mediated FA signaling, leading to changes in cytoskeletal organization. ${ }^{38,39}$ As shown in Figure 4, cells were co-stained for the nucleus (DAPI, blue), actin filaments (red) and vinculin (universal FA marker, green). After a 45-min incubation, cells on the smooth PS and PS NT substrates exhibited different actin filament organization and FA formation. Actin filaments with high density were observed for cells on the smooth PS substrate. Short filopodia were also observed at the edge of cells. The great spreading of cells and the wide distribution of actin and vinculin indicate that cells on the smooth PS substrate had good motility in all directions without topographical sensing. In contrast, cells on the PS NT substrate exhibited actin filaments with low density. The cells spread slightly, while actin and vinculin distributed with polarity. These results indicate that cells respond to the surface nanostructures of the PS NT substrate by topographical sensing through nanoscale molecular arrangement and organization, as demonstrated by the topographical interactions between cell filopodia and substrate nanotubes in Figure $3 \mathrm{f}$ and Supplementary Figure S2b.

\section{Influence of surface modification and surface structures of the PS} NT substrate to cell capture

To investigate the influence of surface modification on cell capture, four types of substrates, the naked substrate and the modified substrates matched to the three modification steps mentioned above, were used to test the cell-capture performance of the PS NT substrates. The results show that non-specific cell adhesion (cells adhering to the substrate through protein or molecule adsorption rather than through specific antigen-antibody recognition) was $\sim 30 \%$ on the naked substrate (Figure $5 \mathrm{j}$ ). After the first (biotinBSA) and second (SA) molecule modification, the non-specific cell adhesion was reduced to $\sim 20 \%$. This reduction was attributed to the utilization of BSA, which reduces non-specific molecular adsorption and cell adhesion. The remaining 20\% non-specific cell adhesion was most likely due to the soft nature of PS that promotes cell attachment and adhesion.

To systematically investigate the influence of substrate structure on cell capture, PS NT substrates with different degrees of aggregation were fabricated by changing the AAO corrosion time. As shown in 

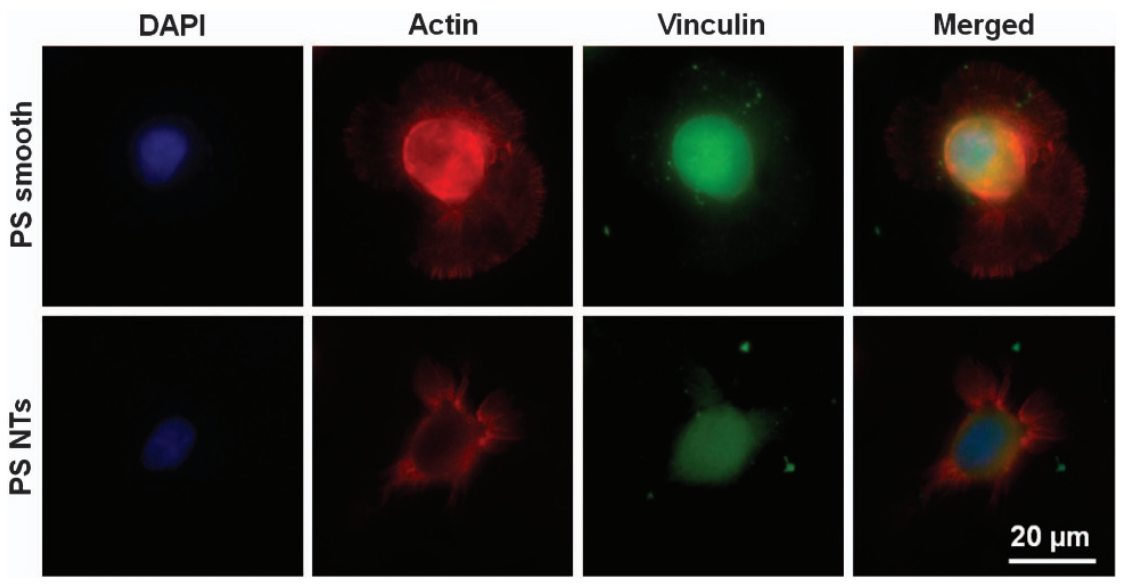

Figure 4 Immunofluorescent staining of cells on smooth polystyrene (PS) and polystyrene nanotube (PS NT) substrates for observation of the action cytoskeleton (red), filopodia and lamellipodia, and focal adhesions (vinculin, green) of cells. The nucleus of cells is stained by 4',6-diamidino2-phenylindol (DAPI).
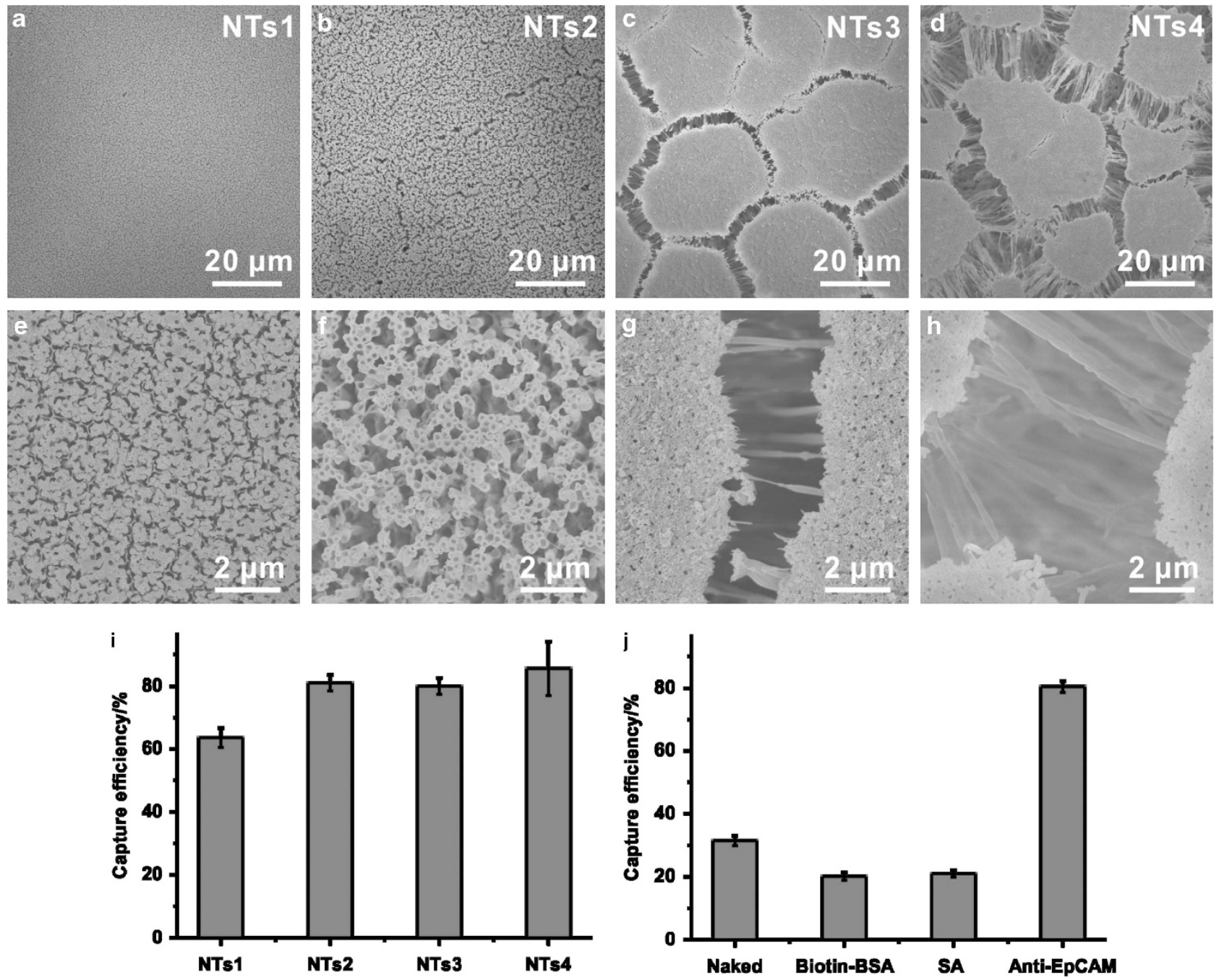

Figure 5 Influence of the substrate surface structures and surface modification on cell capture. (a-d) Polystyrene nanotube (PS NT) substrates with different aggregation degrees. (e-h) Detailed surface structures of four substrates, from NTs1 to NTs4. (i) Along with the increase in the aggregation degree of nanotubes, the cell-capture efficiency increases and reaches the maximum for the NTs2 substrate. (j) The surface modification process reduces the nonspecific cell adhesion of the PS NT substrate. 
Figures $5 \mathrm{a}-\mathrm{d}$, by increasing the corrosion time of AAO from 5 to $15 \mathrm{~min}, 30 \mathrm{~min}$ and $2 \mathrm{~h}$, the exposed PS nanotubes turned from short to long, and the aggregation degree increased gradually from NTs1 to NTs4 (Figures $5 \mathrm{e}-\mathrm{h}$, detailed surface morphologies of these four substrates). The aggregation of PS nanotubes also reflects the soft nature of PS material, in contrast to the previously reported inorganic nanostructures, which are rigid and do not aggregate. ${ }^{22,24,25}$ The cellcapture experiment shows that more cells were captured with the enhancement of the aggregation degree from NTs1 to NTs2 (Figure 5i). Then, the cell-capture efficiency changed only slightly from NTs2 to NTs4, indicating that a large aggregation degree of PS nanotubes does not influence cell capture. The low capture efficiency of the NTs1 substrate was due to the short length of the nanotubes, which reduced the topographical interactions between cells and substrates. (Unless otherwise stated, the PS NT substrates referred to in the text represent PS NTs2.)

Influence of capture time on the specific cell capture and non-specific cell adhesion

To study the influence of capture time on cell-capture efficiency, capture experiments with different times from $5 \mathrm{~min}$ to $2 \mathrm{~h}$ were performed on both the smooth PS and PS NT substrates. The results show that as the capture time increases, more cells are captured on both the smooth and NT substrates (Figure 6a). The cell-capture efficiency of the PS NT substrates reached the maximum value when the time was longer than $30 \mathrm{~min}$. In contrast, after reaching the first equilibrium, the cell-capture numbers of the smooth PS substrate increased again and finally reached a second equilibrium at $\sim 2 \mathrm{~h}$. This phenomenon is also different from the cell-capture results of the previously reported inorganic smooth cell-capture substrates, whose cell-capture numbers usually reached an equilibrium after the first maximum. ${ }^{17,22,34}$ This second increment in cell-capture number was most likely due to the high affinity of the soft PS to cells, which caused an increase in non-specific cell adhesion with increasing incubation time.

To better represent the cell-capture specificity and to optimize the cell-capture performance of PS NT substrates, three non-specific cell lines-Hela (human cervical cancer cell lines) as an adherent epithelial cell control and Jurkat (T-lymphocyte cell line) and Daudi (lymphoma cell lines) as suspension lymphocyte controls-were used for a specific cell-capture contrast experiment. The results show that at the capture time of $45 \mathrm{~min}$, when the specific capture efficiency reached the maximum $(\sim 80 \%)$, non-specific cell adhesion was relatively high: $\sim 30 \%$ of adherent Hela and $10 \%$ of suspension Jurkat and Daudi (Figure 6c). This finding indicates that at this capture condition, although the specific capture efficiency was high, non-specific cell adhesion also occurred abundantly, reducing the capture purity. However, by shortening the capture time to $20 \mathrm{~min}$, the non-specific cell adhesion was reduced significantly (Figure 6c). Hela adhesion reduced to $\sim 7 \%$, and almost no Jurkat and Daudi cells adhered on the substrate, while the specific cell-capture efficiency still retained a very high value $(\sim 70 \%)$. These results indicate that high-efficiency and high-purity cell capture can be achieved on the PS NT substrates in a shorter capture time $(20 \mathrm{~min})$ compared with inorganic cell-capture systems, of which the highest capture performance was reached with a $45 \mathrm{~min}$ or longer capture time. ${ }^{22,24,26}$ This high-efficiency and high-purity cell capture in a shorter capture time is preferred in real-blood capture situations and will facilitate rapid and efficient clinical diagnosis to help save patient lives.

An additional two cell lines (PC3 prostate cancer and T24 bladder cancer cell lines) were used to test whether high capture efficiency at
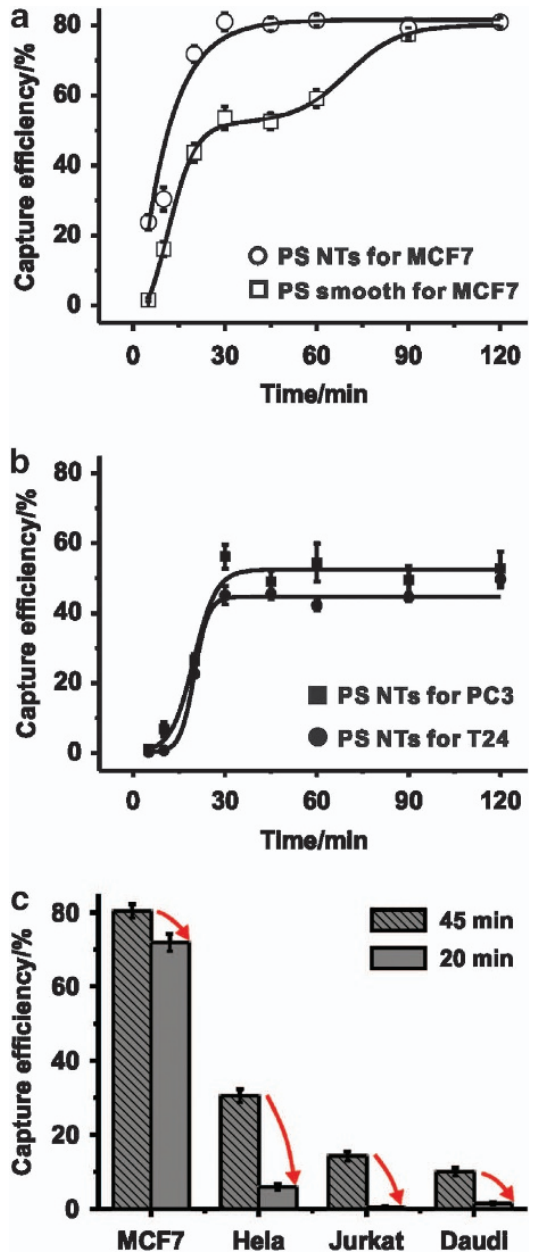

Figure 6 Influence of capture time on the specific cell capture and nonspecific cell adhesion for the polystyrene nanotube (PS NT) substrates. (a) As the capture time increases, the cell-capture efficiency on the PS NT substrate increases gradually and reaches the maximum when the time exceeds $30 \mathrm{~min}$. For the smooth PS substrate, the cell-capture efficiency increases again after the first equilibrium due to non-specific cell adhesion. (b) For another two epithelial cell adhesion molecule (EpCAM)-positive cell lines (PC3 prostate cancer and T24 bladder cancer cell lines), the maximum capture efficiency also reaches the maximum when the time exceeds $30 \mathrm{~min}$. However, the capture efficiency at $20 \mathrm{~min}$ for these two cell lines is comparatively low. (c) When the capture time is shortened from 45 to $20 \mathrm{~min}$, the specific MCF7 cell-capture efficiency decreases slightly and still retains a high value. In contrast, non-specific cell adhesion, especially of the Jurkat and Daudi suspension cell lines, reduces significantly. Four different cells lines: MCF7 (specific) and Hela (non-specific), adherent epithelial cell control; and Jurkat and Daudi (non-specific, suspension), lymphocyte control.

shorter capture time (20 min) is common to all EpCAM-positive cancer cells. The results showed that for both PC3 and T24 cell lines, cell-capture efficiency reached the maximum when the time exceeded $30 \mathrm{~min}$ (Figure 6b). The same occurred with MCF7 cells. The maximum capture efficiency of PC3 was $\sim 50 \%$, and that of T24 was $\sim 45 \%$. However, the cell-capture efficiency for both PC3 and T24 at 20 min was very low and different from the result for MCF7. Thus, for these two cell lines, the capture time can only be shortened to $30 \mathrm{~min}$. Moreover, because the maximum capture efficiency for PC3 and T24 is not very high, the anti-EpCAM-modified PS NT substrate will be more effective for capturing MCF7 cell lines and 


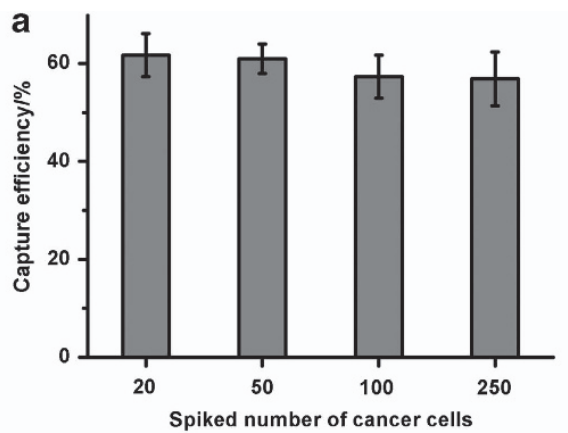

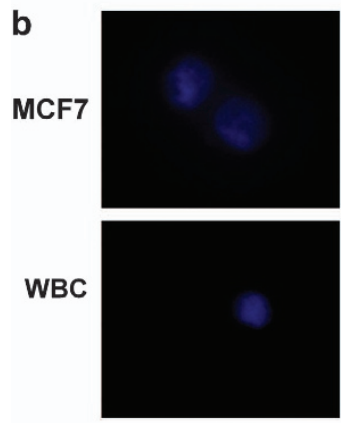

DAPI
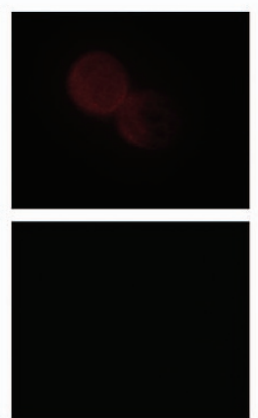

CK

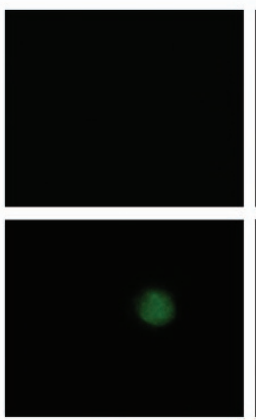

CD45

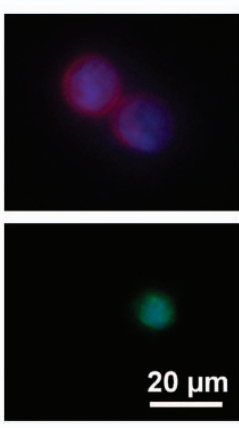

Merged

Figure 7 Capture efficiency of the polystyrene nanotube (PS NT) substrates for artificial whole-blood samples spiked with rare cancer cells. (a) Approximately 60\% capture efficiency is achieved on the PS NT substrates for artificial whole-blood samples with concentrations of 20, 50, 100 and 250 cells per $\mathrm{ml}$. (b) Three-color immunostaining method for identifying target cancer cells (MCF7) from white blood cells (WBC). 4',6-Diamidino-2phenylindol (DAPI) stains the nucleus, PE-labeled anti-cytokeratin (CK, a protein marker for epithelial cells) stains MCF7, and fluorescein isothiocyanate (FITC)-labeled anti-CD45 (a marker for WBCs) stains WBCs.

detecting CTCs of breast cancer patients. The different cell-capture results for MCF7, PC3 and T24 cell lines are most likely due to the different expression quantity of EpCAM on cell surfaces (the mean number of EpCAM molecules per cell: 509500 for MCF7; 51667 for PC3; and 2167 for T24). ${ }^{21,40}$

In addition, the viability of cells captured on the PS NT substrate was tested by the live/dead cell staining method using acridine orange/PI dyes. The results showed that the viability of cells was very high, $\quad 97.7 \%$ (see supporting information, Supplementary Figure S3). This result indicates that the PS NT substrate is very cyto-compatible, which is beneficial for downstream cell culture and biological studies after cell separation.

Cell-capture efficiency of the PS NT substrate for artificial wholeblood samples spiked with rare cancer cells

To further evaluate the cell-capture efficiency of the PS NT substrate in a low-cell-density situation and in blood with rare CTCs, cellcapture experiments were carried out by spiking rare MCF7 cells in blood to mimic real-blood samples. Therefore, 20, 50, 100 and 250 cells pre-stained by $\mathrm{DiD}$ dye (emitting red fluorescence) were separately spiked in $1 \mathrm{ml}$ mouse blood. Then, $1 \mathrm{ml}$ of the as-prepared blood sample was covered on the anti-EpCAM-modified PS NT substrate for a 20-min incubation. After that, the PS NT substrates were gently rinsed with PBS five times and taken for fluorescence imaging. The results show that $\sim 60 \%$ capture efficiency was achieved for spiked cells from whole blood (Figure 7a). Although the capture efficiency was reduced by $\sim 10 \%$ compared with the efficiency when $3 \times 10^{5}$ cells were used at the same capture time $(20 \mathrm{~min})$, it was still very high compared with the efficiencies observed using commercial techniques, ${ }^{20}$ showing the potential application of the PS NT substrate for further clinical sample detection and cancer diagnosis.

For artificial whole-blood samples spiked with MCF7 cells, target cancer cells captured on the substrate can also be identified from nonspecifically adhered white blood cells by a commonly used three-color immunostaining method rather than pre-staining. This three-color immunostaining method, which includes DAPI (for DNA content in the nucleus), PE-labeled anti-CK (a protein marker for epithelial cells) and FITC-labeled anti-CD45 (a marker for WBCs) staining, is suitable for use in clinical patient blood samples to detect CTCs. As shown in Figure $7 \mathrm{~b}$, the combined results were utilized to distinguish CTCs $(\mathrm{DAPI}+/ \mathrm{CK}+/ \mathrm{CD} 45-, 10 \mu \mathrm{m}<$ cell sizes $<40 \mu \mathrm{m})$ from WBCs (DAPI $+/ C K-/ C D 45+$, cell sizes $<15 \mu \mathrm{m})$ and cellular debris.

\section{CONCLUSION}

In conclusion, rapid and highly efficient breast cancer-cell capture from whole-blood samples has been achieved with low non-specific cell adhesion on a soft PS NT substrate with high cell viability by integrating the soft nature of PS with specific capture agents and surface structures, inspired by the cell microenvironment. Although the PS polymer is softer than inorganic materials, it is still stiffer than the tissue where cells exist. Therefore, it is worth investigating the mechanism further by designing substrate materials with variable stiffness or using other types of soft polymers, such as hydrogels, with a stiffness closer to that of the tissue. This proof-of-concept study is expected to provide a potentially optimal candidate for high-quality cancer-cell capture and detection technologies, which will benefit metastasis monitoring, cancer treatment, and other cancer-related biological studies. The influence of softness on cell capture revealed 
here will open up a new avenue for the design of cell-material interfaces for advanced cell-based diagnostic and detection platforms.

\section{CONFLICT OF INTEREST}

The authors declare no conflict of interest.

\section{ACKNOWLEDGEMENTS}

This research is supported by National Research Fund for Fundamental Key Projects (2012CB933800, 2011CB935700, 2012CB933200, 2009CB930404, 2010CB934700), the National Natural Science Foundation (21175140, 20974113, 21121001, 21071148, 20920102036, 91127025), and the Key Research Program of the Chinese Academy of Sciences (KJZD-EW-M01).

1 Schwarz, U. Soft matters in cell adhesion: rigidity sensing on soft elastic substrates. Soft Matter 3, 263-266 (2007)

2 Discher, D. E., Janmey, P. \& Wang, Y. L. Tissue cells feel and respond to the stiffness of their substrate. Science 310, 1139-1143 (2005).

3 Vogel, V. \& Sheetz, M. Local force and geometry sensing regulate cell functions. Nat. Rev. Mol. Cell Bio. 7, 265-275 (2006).

4 Ross, A. M., Jiang, Z., Bastmeyer, M. \& Lahann, J. Physical aspects of cell culture substrates: topography, roughness, and elasticity. Small 8, 336-355 (2012).

5 Pelham, R. J. \& Wang, Y. L. Cell locomotion and focal adhesions are regulated by substrate flexibility. Proc. Natl Acad. Sci. USA 94, 13661-13665 (1997).

6 Engler, A. J., Sen, S., Sweeney, H. L. \& Discher, D. E. Matrix elasticity directs stem cel lineage specification. Cell 126, 677-689 (2006)

7 Trappmann, B., Gautrot, J. E., Connelly, J. T., Strange, D. G. T., Li, Y., Oyen, M. L. Stuart, M. A. C., Boehm, H., Li, B. J., Vogel, V., Spatz, J. P., Watt, F. M. \& Huck, W. T. S. Extracellular-matrix tethering regulates stem-cell fate. Nat. Mater. 11, 642-649 (2012).

8 Discher, D. E., Mooney, D. J. \& Zandstra, P. W. Growth factors, matrices, and force combine and control stem cells. Science 324, 1673-1677 (2009).

9 Paszek, M. J., Zahir, N., Johnson, K. R., Lakins, J. N., Rozenberg, G. I., Gefen, A., Reinhart-King, C. A., Margulies, S. S., Dembo, M., Boettiger, D., Hammer, D. A. \& Weaver, V. M. Tensional homeostasis and the malignant phenotype. Cancer Cell 8, 241-254 (2005)

10 Levental, I., Georges, P. C. \& Janmey, P. A. Soft biological materials and their impact on cell function. Soft Matter 3, 299-306 (2007).

11 Flanagan, L. A., Ju, Y. E., Marg, B., Osterfield, M. \& Janmey, P. A. Neurite branching on deformable substrates. Neuroreport 13, 2411-2415 (2002).

12 Engler, A. J., Griffin, M. A., Sen, S., Bonnetnann, C. G., Sweeney, H. L. \& Discher, D. E. Myotubes differentiate optimally on substrates with tissue-like stiffness: pathological implications for soft or stiff microenvironments. J. Cell Biol. 166 , 877-887 (2004).

13 Tirrell, M., Kokkoli, E. \& Biesalski, M. The role of surface science in bioengineered materials. Surf. Sci. 500, 61-83 (2002).

14 Lee, K. Y. \& Mooney, D. J. Hydrogels for tissue engineering. Chem. Rev. 101, 1869-1879 (2001)

15 Shalek, A. K., Robinson, J. T., Karp, E. S., Lee, J. S., Ahn, D.-R., Yoon, M.-H., Sutton, A., Jorgolli, M., Gertner, R. S., Gujral, T. S., MacBeath, G., Yang, E. G. \& Park, H. Vertica silicon nanowires as a universal platform for delivering biomolecules into living cells. Proc. Natl Acad. Sci. USA 107, 1870-1875 (2010).

16 Fischer, K. E., Aleman, B. J., Tao, S. L., Daniels, R. H., Li, E. M., Bunger, M. D. Nagaraj, G., Singh, P., Zettl, A. \& Desai, T. A. Biomimetic nanowire coatings for next generation adhesive drug delivery systems. Nano Lett. 9, 716-720 (2009).

17 Chen, L., Liu, X. L., Su, B., Li, J., Jiang, L., Han, D. \& Wang, S. T. Aptamer-mediated efficient capture and release of T lymphocytes on nanostructured surfaces. Adv. Mater. 23, 4376-4380 (2011)

18 Kim, S. T., Kim, D. J., Kim, T. J., Seo, D. W., Kim, T. H., Lee, S. Y., Kim, K., Lee, K. M. \& Lee, S. K. Novel streptavidin-functionalized silicon nanowire arrays for CD4 $(+) T$ lymphocyte separation. Nano Lett. 10, 2877-2883 (2010).

19 Hughes, A. D. \& King, M. R. Nanobiotechnology for the capture and manipulation of circulating tumor cells. WIREs Nanomed. Nanobi. 4, 291-309 (2012).

20 Pantel, K., Brakenhoff, R. H. \& Brandt, B. Detection, clinical relevance and specific biological properties of disseminating tumour cells. Nat. Rev. Cancer 8, 329-340 (2008).

21 Nagrath, S., Sequist, L. V., Maheswaran, S., Bell, D. W., Irimia, D., Ulkus, L., Smith, M. R. Kwak, E. L., Digumarthy, S., Muzikansky, A., Ryan, P., Balis, U. J., Tompkins, R. G.,
Haber, D. A. \& Toner, M. Isolation of rare circulating tumour cells in cancer patients by microchip technology. Nature 450, 1235-U1210 (2007).

22 Wang, S. T., Wang, H., Jiao, J., Chen, K. J., Owens, G. E., Kamei, K. I., Sun, J., Sherman, D. J., Behrenbruch, C. P., Wu, H. \& Tseng, H. R. Three-dimensional nanostructured substrates toward efficient capture of circulating tumor cells. Angew. Chem. Int. Ed. 48, 8970-8973 (2009).

23 Wang, S. T., Liu, K., Liu, J. A., Yu, Z. T. F., Xu, X. W., Zhao, L. B., Lee, T., Lee, E. K., Reiss, J., Lee, Y. K., Chung, L. W. K., Huang, J. T., Rettig, M., Seligson, D. Duraiswamy, K. N., Shen, C. K. F. \& Tseng, H. R. Highly efficient capture of circulating tumor cells by using nanostructured silicon substrates with integrated chaotic micromixers. Angew. Chem. Int. Ed. 50, 3084-3088 (2011).

24 Park, G.-S., Kwon, H., Kwak, D. W., Park, S. Y., Kim, M., Lee, J.-H., Han, H., Heo, S. Li, X. S., Lee, J. H., Kim, Y. H., Lee, J.-G., Yang, W., Cho, H. Y., Kim, S. K. \& Kim, K. Full surface embedding of gold clusters on silicon nanowires for efficient capture and photothermal therapy of circulating tumor cells. Nano Lett. 12, 1638-1642 (2012).

25 Lee, S. K., Kim, G. S., Wu, Y., Kim, D. J., Lu, Y., Kwak, M., Han, L., Hyung, J. H., Seol, J. K., Sander, C., Gonzalez, A., Li, J. \& Fan, R. Nanowire substrate-based laser scanning cytometry for quantitation of circulating tumor cells. Nano Lett. 12, 2697-2704 (2012)

26 Zhang, N. A., Deng, Y. L., Tai, Q. D., Cheng, B. R., Zhao, L. B., Shen, Q. L., He, R. X. Hong, L. Y., Liu, W., Guo, S. S., Liu, K., Tseng, H. R., Xiong, B. \& Zhao, X. Z. Electrospun $\mathrm{TiO}_{2}$ nanofiber-based cell capture assay for detecting Circulating tumor cells from colorectal and gastric cancer patients. Adv. Mater. 24, 2756-2760 (2012).

27 Sekine, J., Luo, S.-C., Wang, S. T., Zhu, B., Tseng, H.-R. \& Yu, H.-h. Functionalized conducting polymer nanodots for enhanced cell capturing: the synergistic effect of capture agents and nanostructures. Adv. Mater. 23, 4788-4792 (2011).

28 Sun, T. L., Qing, G. Y., Su, B. L. \& Jiang, L. Functional biointerface materials inspired from nature. Chem. Soc. Rev. 40, 2909-2921 (2011).

29 Sun, T. L., Feng, L., Gao, X. F. \& Jiang, L. Bioinspired surfaces with special wettability. Acc. Chem. Res. 38, 644-652 (2005).

30 Liu, X. L., Zhou, J., Xue, Z. X., Gao, J., Meng, J. X., Wang, S. T. \& Jiang, L. Clam's shell inspired high-energy inorganic coatings with underwater low adhesive superoleophobicity. Adv. Mater. 24, 3401-3405 (2012).

31 Liu, X. L., Gao, J., Xue, Z. X., Chen, L., Lin, L., Jiang, L. \& Wang, S. T. Bioinspired oil strider floating at the oil/water interface supported by huge superoleophobic force. ACS Nano 6, 5614-5620 (2012).

32 Jin, M. H., Feng, X. J., Feng, L., Sun, T. L., Zhai, J., Li, T. J. \& Jiang, L. Superhydrophobic aligned polystyrene nanotube films with high adhesive force. Adv. Mater. 17, 1977-1981 (2005).

33 Went, P. T., Lugli, A., Meier, S., Bundi, M., Mirlacher, M., Sauter, G. \& Dirnhofer, S Frequent EpCam protein expression in human carcinomas. Hum. Pathol. 35, 122-128 (2004).

34 Liu, H. L., Liu, X. L., Meng, J. X., Zhang, P. C., Yang, G., Su, B., Sun, K., Chen, L., Han, D. Wang, S. T. \& Jiang, L. Hydrophobic interaction-mediated capture and release of cance cells on thermoresponsive nanostructured surfaces. Adv. Mater. 25, 922-927 (2013).

35 Huang, T. T., Sturgis, J., Gomez, R., Geng, T., Bashir, R., Bhunia, A. K., Robinson, J. P. \& Ladisch, M. R. Composite surface for blocking bacterial adsorption on protein biochips. Biotechnol. Bioeng. 81, 618-624 (2003).

36 Yang, D. Y., Niu, X., Liu, Y. Y., Wang, Y., Gu, X., Song, L. S., Zhao, R., Ma, L. Y., Shao, Y. M. \& Jiang, X. Y. Electrospun nanofibrous membranes: a novel solid substrate for microfluidic immunoassays for HIV. Adv. Mater. 20, 4770-4775 (2008).

37 Sorelli, L., Constantinides, G., Ulm, F. J. \& Toutlemonde, F. The nano-mechanical signature of ultra high performance concrete by statistical nanoindentation techniques. Cement Concrete. Res. 38, 1447-1456 (2008).

38 Yim, E. K. F., Darling, E. M., Kulangara, K., Guilak, F. \& Leong, K. W. Nanotopographyinduced changes in focal adhesions, cytoskeletal organization, and mechanical properties of human mesenchymal stem cells. Biomaterials 31, 1299-1306 (2010).

39 Chen, W. Q., Weng, S. N., Zhang, F., Allen, S., Li, X., Bao, L. W., Lam, R. H. W. Macoska, J. A., Merajver, S. D. \& Fu, J. P. Nanoroughened surfaces for efficient capture of circulating tumor cells without using capture antibodies. ACS Nano 7, 566-575 (2013).

40 Rao, C. G., Chianese, D., Doyle, G. V., Miller, M. C., Russell, T., Sanders, R. A. \& Terstappen, L. W. M. M. Expression of epithelial cell adhesion molecule in carcinoma cells present in blood and primary and metastatic tumors. Int. J. Oncol. 27, 49-57 (2005)

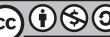

This work is licensed under a Creative Commons Attribution-NonCommercial-ShareAlike 3.0 Unported License. To view a copy of this license, visit http://creativecommons. org/licenses/by-nc-sa/3.0/

Supplementary Information accompanies the paper on the NPG Asia Materials website (http://www.nature.com/am) 